\title{
Article \\ Epithelial Regeneration Ability of Crohn's Disease Assessed Using Patient-Derived Intestinal Organoids
}

\author{
Chansu Lee ${ }^{1,2} \oplus$, Sung-Noh Hong ${ }^{1,2, *}$, Eun-Ran Kim ${ }^{1}$, Dong-Kyung Chang ${ }^{1}$ and Young-Ho Kim ${ }^{1}$ \\ 1 Samsung Medical Center, Department of Medicine, Sungkyunkwan University School of Medicine, \\ 81 Irwon-ro, Gangnam-gu, Seoul 06351, Korea; cslhero@gmail.com (C.L.); er.kim@samsung.com (E.-R.K.); \\ do.chang@samsung.com (D.-K.C.); bowelkim@gmail.com (Y.-H.K.) \\ 2 Stem Cell \& Regenerative Medicine Center, Samsung Medical Center, 81 Irwon-ro, \\ Gangnam-gu, Seoul 06351, Korea \\ * Correspondence: sungnoh.hong@samsung.com or gisnhong@gmail.com; Tel.: +82-2-3410-3409; \\ Fax: +82-2-3410-6983
}

Citation: Lee, C.; Hong, S.-N.; Kim, E.-R.; Chang, D.-K.; Kim, Y.-H. Epithelial Regeneration Ability of Crohn's Disease Assessed Using Patient-Derived Intestinal Organoids. Int. J. Mol. Sci. 2021, 22, 6013. https://doi.org/10.3390/ijms22116013

Academic Editors: Guillermo Gomez and Oliver Sieber

Received: 12 May 2021

Accepted: 31 May 2021

Published: 2 June 2021

Publisher's Note: MDPI stays neutral with regard to jurisdictional claims in published maps and institutional affiliations.

Copyright: () 2021 by the authors. Licensee MDPI, Basel, Switzerland. This article is an open access article distributed under the terms and conditions of the Creative Commons Attribution (CC BY) license (https:// creativecommons.org/licenses/by/ $4.0 /)$.
Abstract: Little is known about the ability for epithelial regeneration and wound healing in patients with inflammatory bowel diseases. We evaluated the epithelial proliferation and wound healing ability of patients with Crohn's disease (CD) using patient-derived intestinal organoids. Human intestinal organoids were constructed in a three-dimensional intestinal crypt culture of enteroscopic biopsy samples from controls and CD patients. The organoid-forming efficiency of ileal crypts derived from CD patients was reduced compared with those from control subjects $(p<0.001)$. Long-term cultured organoids ( $\geq 6$ passages) derived from controls and CD patients showed an indistinguishable microscopic appearance and culturing behavior. Under TNF $\alpha$-enriched conditions $(30 \mathrm{ng} / \mathrm{mL})$, the organoid reconstitution rate and cell viability of $\mathrm{CD}$ patient-derived organoids were significantly lower than those of the control organoids ( $p<0.05$ for each). The number of EdU+cells was significantly lower in TNF $\alpha$-treated organoids derived from CD patients than in TNF $\alpha$-treated control organoids $(p<0.05)$. In a wound healing assay, the unhealed area in TNF $\alpha$-treated CD patientderived organoids was significantly larger than that of TNF $\alpha$-treated control organoids $(p<0.001)$. The wound healing ability of CD patient-derived organoids is reduced in TNF $\alpha$-enriched conditions, due to reduced cell proliferation. Epithelial regeneration ability may be impaired in patients with $C D$.

Keywords: Crohn's disease; tumor necrosis factor-alpha; intestinal organoids; epithelial regeneration; wound healing

\section{Introduction}

The intestinal epithelium, which acts as a frontline defense of the human body, is repetitively injured and continuously regenerated [1]. Epithelial regeneration depends on the self-renewal and proliferation of LGR5+ intestinal stem cells (ISCs), which occur in intestinal crypts [2]. Crohn's disease (CD) is a chronic relapsing-remitting inflammatory bowel disease (IBD), characterized by cycles of mucosal inflammation and ulceration, followed by regeneration and restoration [3]. Impaired epithelial regeneration can lead to sustained intestinal inflammation and can be accompanied by ulcers and complications, such as fibrosis and fistulas.

Nevertheless, little is known about the ability for epithelial regeneration and wound healing in patients with CD. Clinical trials could not be implemented to evaluate this issue due to ethical reasons and clinical heterogeneity. Classical tumor-derived cell lines and animal model systems have inherent limitations [4]. Immortalized cell lines consist of a homogenous cellular component and evade cellular senescence, due to the presence of certain mutations [5]. Animal models have limitations in replicating human specific biologic processes [6]. With the advent of intestinal epithelium-derived organoids, it is 
possible to cultivate all epithelial cellular components and re-create the functional cryptvillus architecture [7-9]; patient-derived intestinal organoids might be appropriate for studying the regenerative ability of epithelial cells. This study aimed to evaluate the epithelial regenerative ability of $\mathrm{CD}$ patient-derived intestinal organoids, compared to that of control subject-derived intestinal organoids.

Inflammation challenges epithelial integrity and barrier function. The intestinal epithelium needs to adapt to a multitude of signals in order to perform the complex process of maintenance and restitution of its barrier function [10]. Tumor necrosis factor-alpha $(\mathrm{TNF} \alpha)$ acts as a major pro-inflammatory and tissue damage-promoting effector during the pathogenesis of $\mathrm{CD}$; this is supported by evidence provided by studies involving experimental mouse models and the therapeutic effects of TNF $\alpha$-neutralizing reagents in IBD treatment [11-13]. Recently, mucosal healing has been considered a therapeutic target, as it improves the prognosis of patients with CD [14]. We aimed to understand the $\mathrm{TNF} \alpha$-induced alteration of epithelial regenerative ability in CD patient-derived organoids compared to that of control organoids.

\section{Results}

Intestinal crypts isolated from duodenal (CD, $n=5$; controls, $n=5)$, jejunal $(\mathrm{CD}, n=9$; controls, $n=13$ ), ileal (CD, $n=43$; controls, $n=15)$, and colonic biopsy samples (CD, $n=12$; controls, $n=8)$ from controls $(n=34)$ and patients with CD $(n=51)$ were cultured in Matrigel with maintenance medium and then organoid-forming efficiency was evaluated. Patient characteristics for the enrolled patients are listed in Table 1. In both groups, the organoid-forming efficiency of duodenal crypts was the highest, followed by jejunal, ileal, and colonic crypts. The organoid-forming efficiency of the ileal crypts obtained from patients with CD was significantly lower than that of the ileal crypts obtained from controls on day $3(53.4 \% \pm 4.0 \%$ vs. $39.6 \% \pm 2.4 \%, p=0.005)$, day $5(38.9 \% \pm 3.0 \%$ vs. $21.1 \% \pm 1.7 \%$, $p<0.001)$, and day $7(29.2 \% \pm 2.8 \%$ vs. $15.7 \% \pm 1.5 \%, p<0.001)$. The organoid-forming efficiency on day 7 of the jejunal crypts obtained from CD patients was numerically lower than that of those obtained from controls ( $46.8 \pm 2.6 \%$ vs. $38.1 \% \pm 3.8 \%$, Figure 1$)$.

Table 1. Characteristics of the enrolled patients, and the number of attempts at constructing organoids.

\begin{tabular}{|c|c|c|c|}
\hline $\begin{array}{l}\text { Total Number of Enrolled } \\
\text { Patients }\end{array}$ & 85 & Number of Attempts to Construct Organoids & 110 * \\
\hline Patients with $C D, n$ & 51 & Number of sub-culture ( $\geq$ passage 3 ) enabled organoids & 61 \\
\hline Age, years (mean \pm S.D) & $36.2 \pm 13.4$ & $\begin{array}{l}\text { CD-patient derived organoids ( } \mathrm{n} \text { of attempts } / \mathrm{n} \text { of } \\
\text { subculture) }\end{array}$ & $69 / 37$ \\
\hline Male, $\mathrm{n}$ & 43 & Duodenal organoids ( $\mathrm{n}$ of attempts/n of subculture) & $5 / 4$ \\
\hline Montreal classification, $\mathrm{n}$ & & Mucosal healing or no duodenal involvement & $5 / 4$ \\
\hline Age at diagnosis $(\mathrm{A} 1 / \mathrm{A} 2 / \mathrm{A} 3)$ & $0 / 42 / 9$ & Active ulcer & $0 / 0$ \\
\hline Location (L1/L2/L3) & $1 / 34 / 16$ & Jejunal organoids ( $\mathrm{n}$ of attempts/n of subculture) & $9 / 8$ \\
\hline Behavior (B1/B2/B3) & $20 / 27 / 4$ & Mucosal healing or no jejunal involvement & $4 / 4$ \\
\hline IBD Medication at sampling, $\mathrm{n}$ & & Active ulcer & $5 / 4$ \\
\hline None & 6 & Ileal organoids (n of attempts/n of subculture) & $43 / 23$ \\
\hline Immunomodulator & 25 & Mucosal healing or no ileal involvement & $8 / 5$ \\
\hline Anti-TNF $\alpha$ & 25 & Active ulcer & $35 / 18$ \\
\hline Sampling modality, $\mathrm{n}$ & & Colonic organoids ( $\mathrm{n}$ of attempts/n of subculture) & $12 / 2$ \\
\hline Colonoscopy & 11 & Mucosal healing or no colonic involvement & $4 / 1$ \\
\hline Single-balloon enteroscopy & 40 & Active ulcer & $8 / 1$ \\
\hline Controls, $\mathrm{n}$ & 34 & Control organoids (n of attempts/n of subculture) & $41 / 24$ \\
\hline Age, years (mean \pm S.D) & $50.6 \pm 17.9$ & Duodenal organoids ( $\mathrm{n}$ of attempts/n of subculture) & $5 / 4$ \\
\hline \multirow[t]{3}{*}{ Male sex, $\mathrm{n}$} & 24 & Jejunal organoids (n of attempts/n of subculture) & $13 / 11$ \\
\hline & & Ileal organoids ( $\mathrm{n}$ of attempts/n of subculture) & $15 / 8$ \\
\hline & & Colonic organoids ( $\mathrm{n}$ of attempts/n of subculture) & $8 / 1$ \\
\hline
\end{tabular}

* Twenty-one patients having samples from two different sites and two patients having samples from three different sites. 


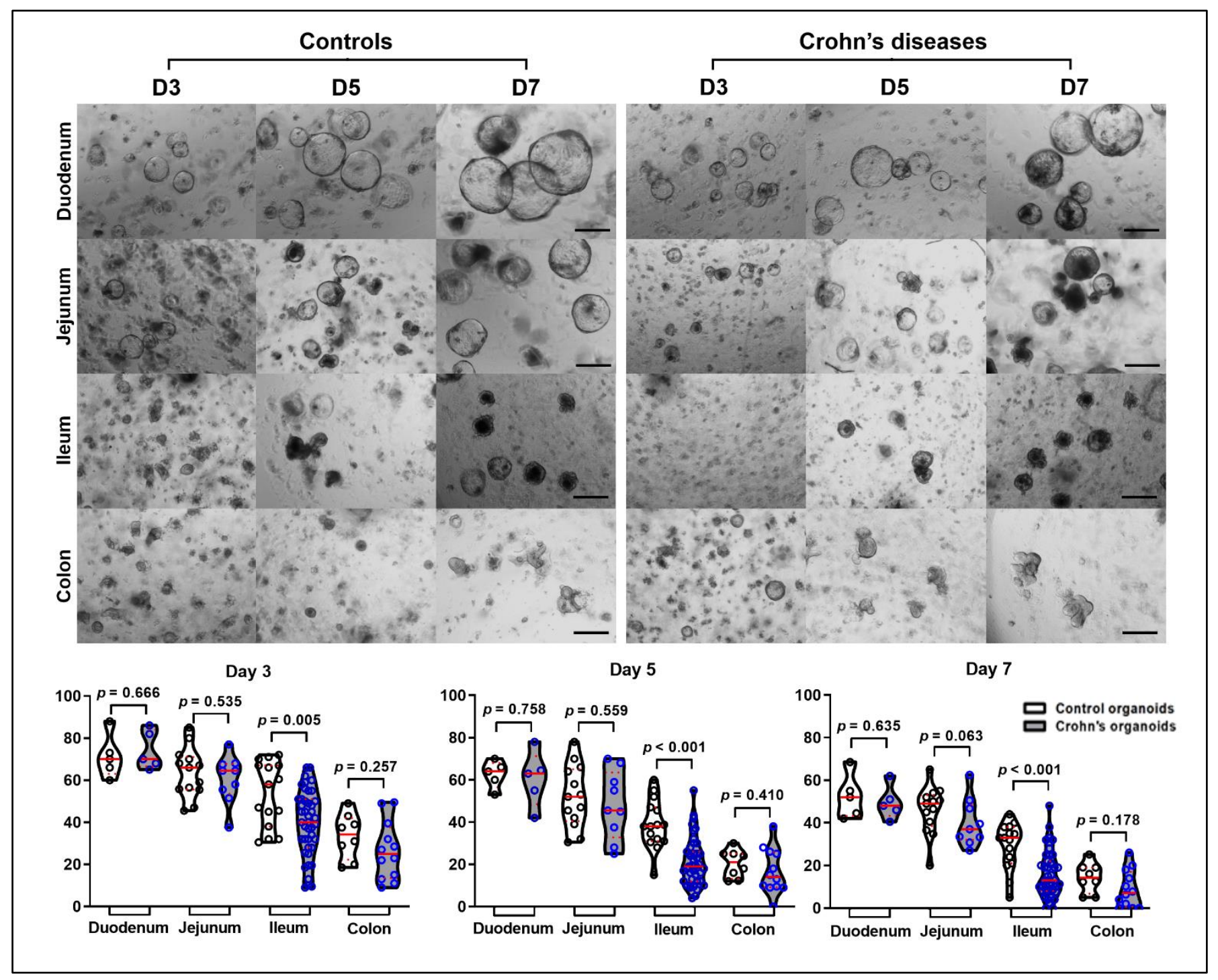

Figure 1. Organoid-forming efficiency. Intestinal crypts were isolated from the duodenum $(n=5)$, jejunum $(n=9)$, ileum $(n=43)$, and colon $(n=12)$ of patients with CD and the duodenum $(n=5)$, jejunum $(n=13)$, ileum $(n=15)$, and colon $(n=8)$ of controls. Differences in organoid-forming efficiency between controls and CD patients based on the location of GI tract were assessed by $t$-test; Scale bar $=200 \mu \mathrm{m}$.

Organoids grown from intestinal crypts were sub-cultured in maintenance medium and the control organoids became uniformly spheroid ( $>90 \%$ of total organoids) after 2-4 passages. However, organoids derived from patients with CD had both enteroid and spheroid forms in the early passages and became uniformly spheroid after 4-6 passages. After six passages, the organoids derived from the controls and patients with CD exhibited consistent spheroid features and culturing behaviors (Figure 2). 


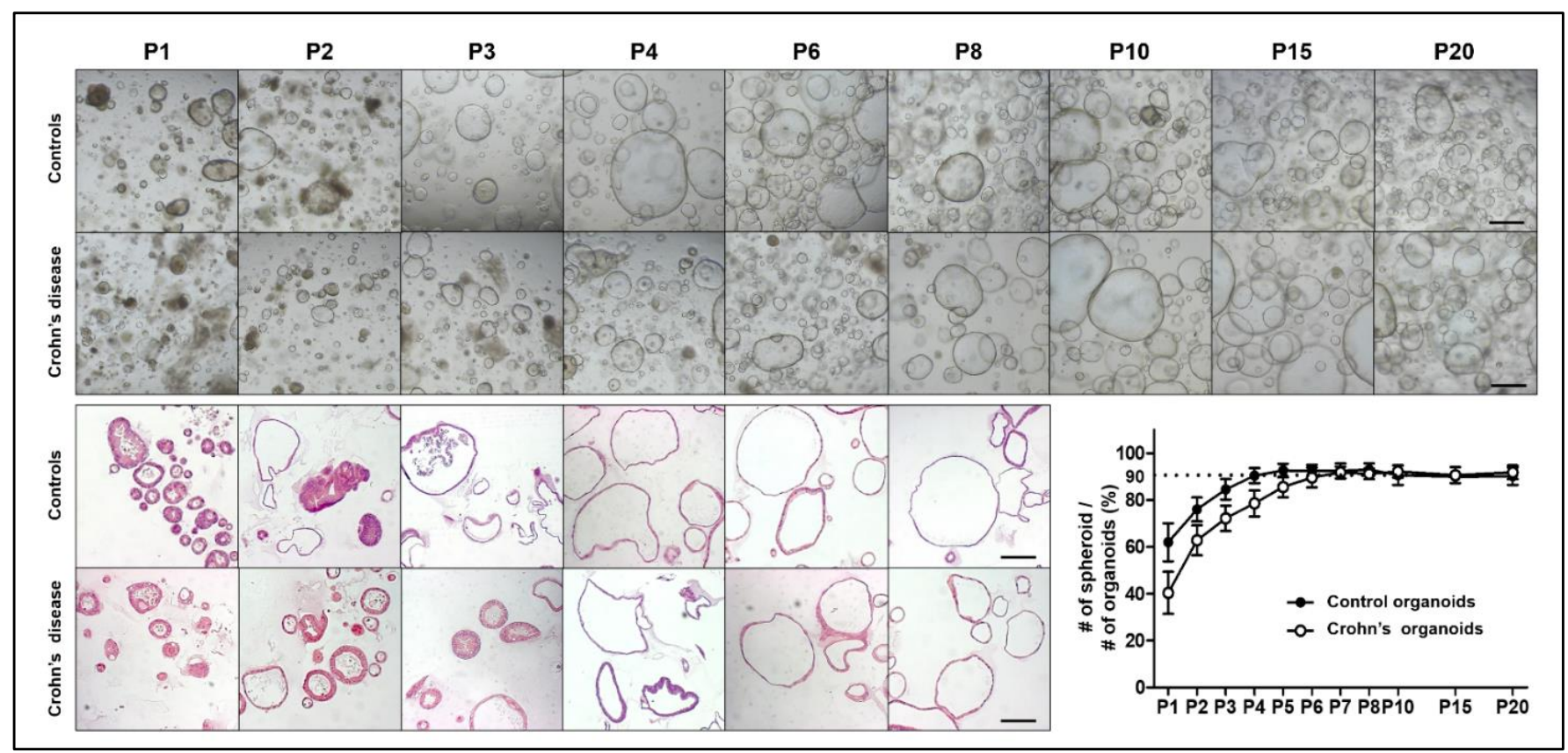

Figure 2. Long-term culture of control and CD patient-derived organoids. Bright field and H\&E staining images of control and $C D$ patient-derived organoids according to the passages. After six passages, the morphology of control and CD patient-derived organoids became identical. Scale bar $=200 \mu \mathrm{m}$.

The shapes of the organoids cultured in the maintenance medium were similar regardless of their location; however, those cultured in differentiation medium tended to have different shapes, depending on their origin. The murine enteroids tended to have more budding structures compared to human enteroids. Ileal organoids typically exhibited budding, whereas jejunal organoids formed thick-walled structures (Figure 3). Previous studies have noted that the cytotoxicity of intestinal organoids occurred in a concentrationdependent manner in response to TNF $\alpha[15,16]$. To address the appropriate concentration of TNF $\alpha$ and the interval of administration to assess the epithelial regenerative ability, control organoids were tested for changes in the expression of the ISC and progenitor marker after TNF $\alpha$ treatment and organoid survival at the various concentrations of TNF $\alpha$. The organoid viability, measured using MTT and the enteroid/spheroid ratio, decreased significantly with a gradual increase in the TNF $\alpha$ concentration. At TNF $\alpha$ concentrations of $\leq 10 \mathrm{ng} / \mathrm{mL}$ in the differentiation medium, changes in cell viability and morphology were negligible; however, the changes observed at TNF $\alpha$ concentrations of $\geq 30 \mathrm{ng} / \mathrm{mL}$ were notable (Figure S1). The expression of LGR5 (active ISC), BMI1 (reserve ISC), HES1 (absorptive progenitor), and ATOH1 (secretory progenitor) increased within $24 \mathrm{~h}$ of TNF $\alpha$ treatment and then decreased (Figure 4). 


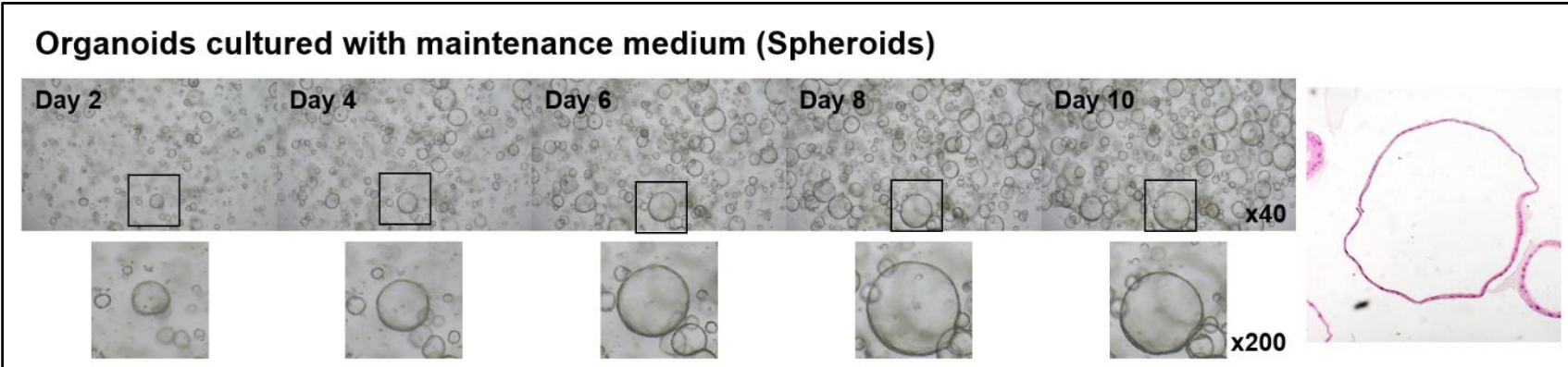

Jejunal organoids cultured with differentiation medium (Enteroids)
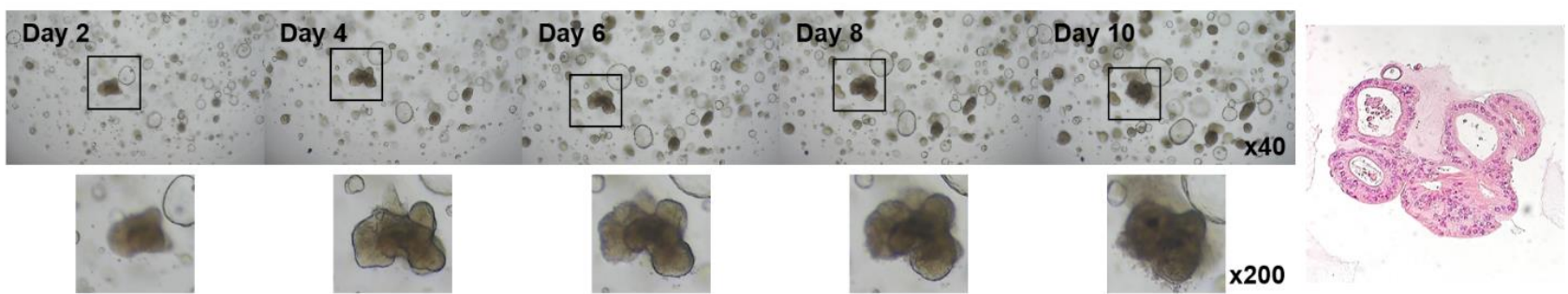

Ileal organoids cultured with differentiation medium (Enteroids)
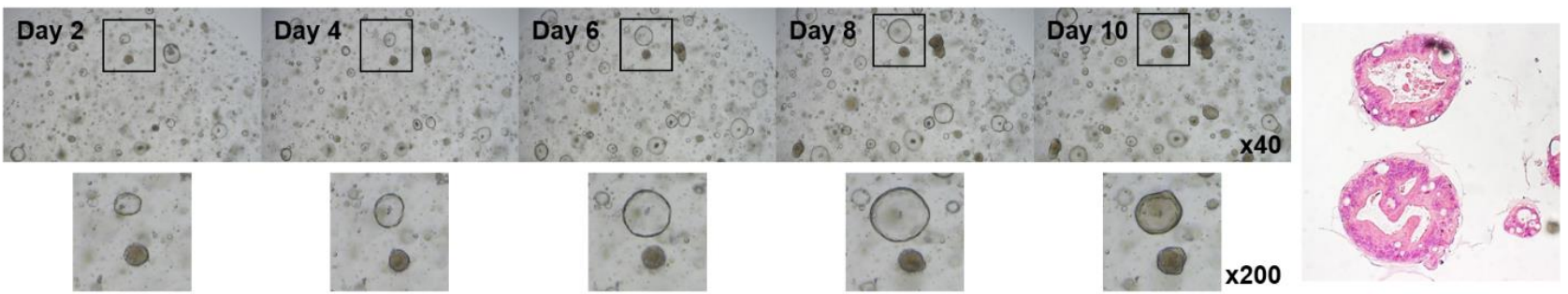

\section{Colonic organoids cultured with differentiation medium (Colonoids)}

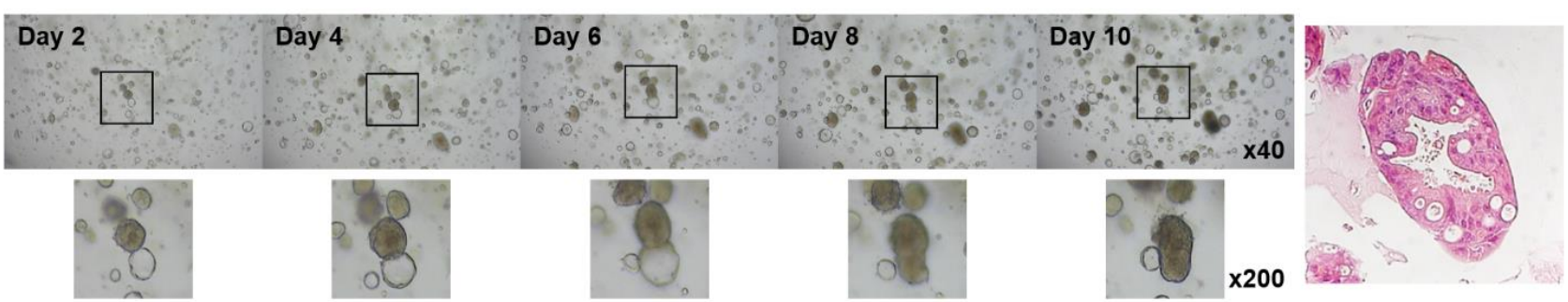

Mouse Enteroids (Mouse small bowel organoids cultured with differentiation media)

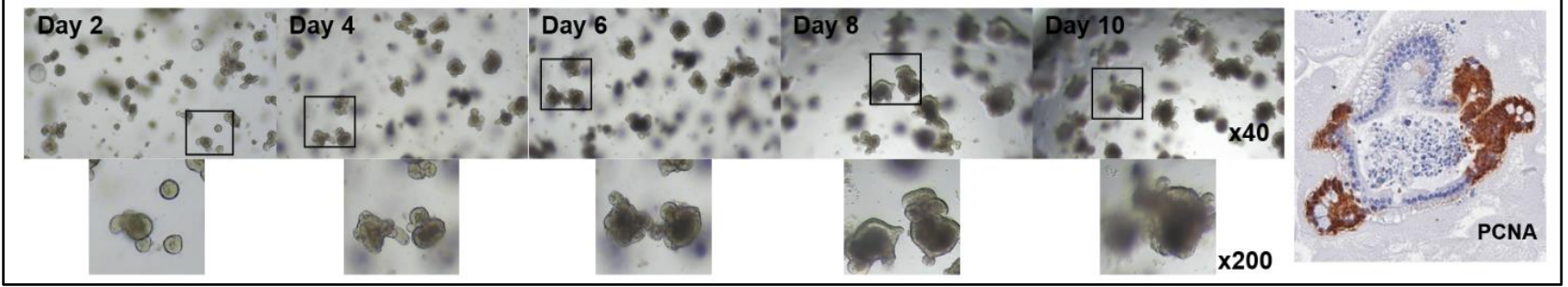

Figure 3. Microscopic appearance of jejunal, ileal, and colonic organoids cultured in differentiation medium. The control organoids cultured in the maintenance medium formed spheroids, while those cultured in the differentiation medium formed enteroids. Jejunal organoids had budding structures, while the ileal and colonial organoids formed thick-walled structures. 


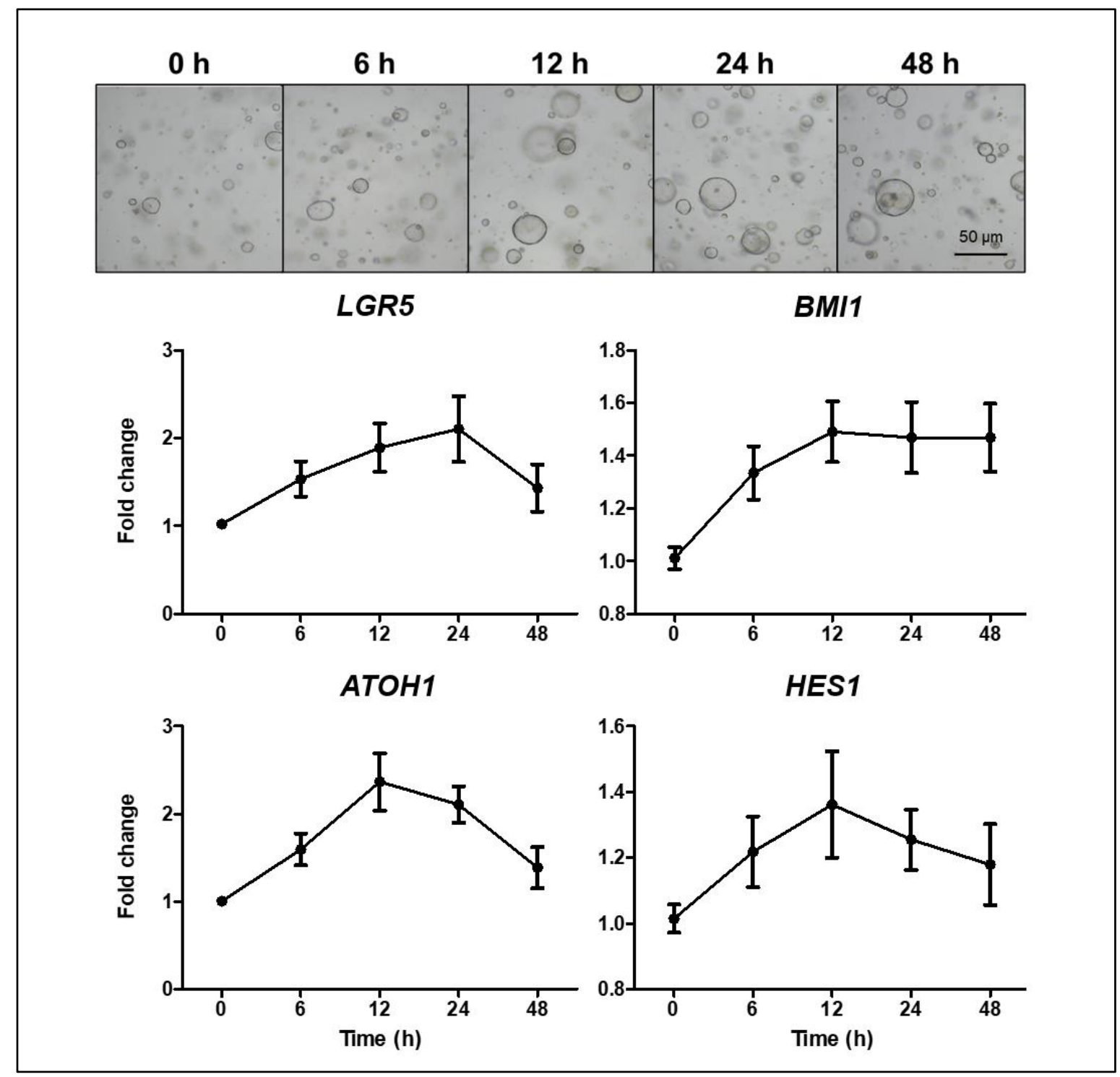

Figure 4. Changes in the expression of LGR5, BMI1, ATHO1, and HES1 in control organoids at 6, 12, 24, and 48 h after treatment with $\mathrm{TNF} \alpha(30 \mathrm{ng} / \mathrm{mL})$. Quantitative reverse transcription polymerase chain reaction was performed with control organoids $(n=3)$ in triplicate.

Based on these results, organoids derived from controls and $\mathrm{CD}$ patients, cultured over the long-term ( $\geq 6$ passages), were treated with $30 \mathrm{ng} / \mathrm{mL}$ TNF $\alpha$ every $24 \mathrm{~h}$ for 10 days (Figure 5A). The epithelial regenerative ability of intestinal organoids was evaluated using organoid reconstitution, MTT, EdU, and wound healing assays. The organoid reconstitution rate of $\mathrm{TNF} \alpha$-treated organoids was significantly lower than that of $\mathrm{TNF} \alpha$ free organoids (jejunal organoids: $68.9 \% \pm 12.4 \%$ vs. $47.4 \% \pm 13.6 \%, p<0.001$; ileal organoids: $55.2 \% \pm 12.3 \%$ vs. $32.8 \% \pm 10.1 \%, p<0.001$ ). There was no significant difference in the organoid reconstitution rate between $\mathrm{TNF} \alpha$-free controls and CD patient-derived organoids; however, the organoid reconstitution rate of $\mathrm{TNF} \alpha$-treated CD patient-derived organoids was significantly lower than that of $\mathrm{TNF} \alpha$-treated control organoids (jejunal organoids: $55.5 \% \pm 11.5 \%$ vs. $39.3 \% \pm 10.6 \%, p=0.011$; ileal organoids: $40.2 \% \pm 6.9 \%$ vs. $25.3 \% \pm 6.6 \%, p=0.027$; Figure $5 \mathrm{~B}, \mathrm{C})$. 


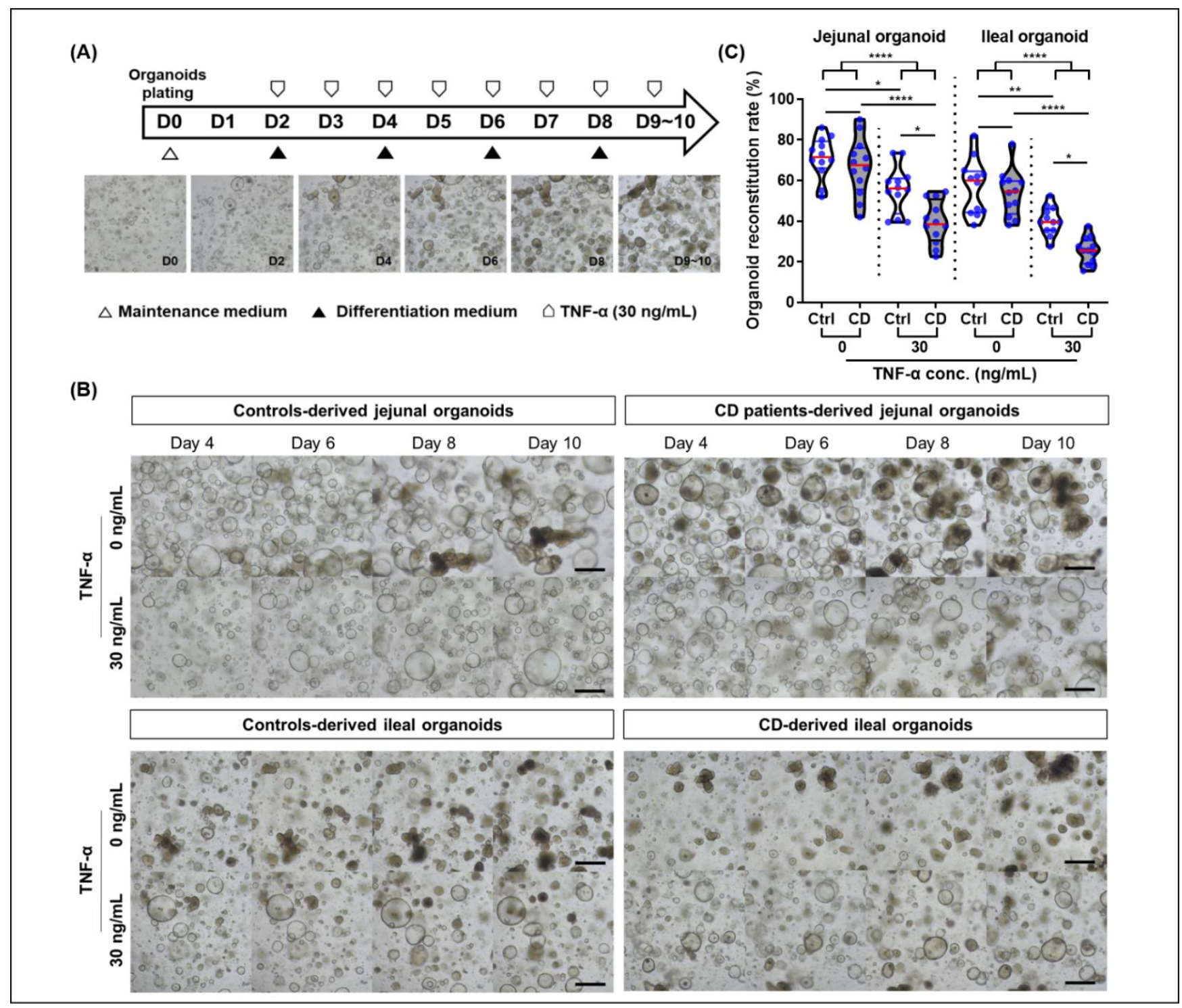

Figure 5. Organoid reconstitution assay in a tumor necrosis factor-alpha (TNF $\alpha$ ) enriched condition. (A) Study flow diagram. (B) Organoid reconstitution assay of jejunal and ileal organoids derived from controls and CD patients $(n=5$ each) in a $\mathrm{TNF} \alpha$-enriched condition. Assays were conducted in triplicate. (C) Organoid reconstitution rate. Reconstituted organoid number is expressed as a percentage value, based on values of TNF $\alpha$-free jejunal organoids derived from controls. Differences were evaluated using ANOVA with Bonferroni's multiple comparison test; ${ }^{*} p<0.05,{ }^{* *} p<0.01$ and ${ }^{* * *} p<0.0001$.

The organoid viability was assessed using MTT; the results showed that the formazan absorbance values of viable TNF $\alpha$-treated organoids were significantly lower than those of $\mathrm{TNF} \alpha$-free organoids (OD of jejunal organoids: $0.96 \pm 0.16$ vs. $0.71 \pm 0.12, p<0.001$; OD of ileal organoids: $0.83 \pm 0.16$ vs. $0.56 \pm 0.16, p<0.001)$. In the TNF $\alpha$-enriched condition, the viable cells of jejunal and ileal CD patient-derived organoids were significantly decreased compared with those of the control organoids (OD of jejunal organoids: $0.79 \pm 0.10 \mathrm{vs}$. $0.62 \pm 0.07, p=0.135$; OD of ileal organoids: $0.66 \pm 0.13$ vs. $0.45 \pm 0.10, p=0.019$; Figure 6). 


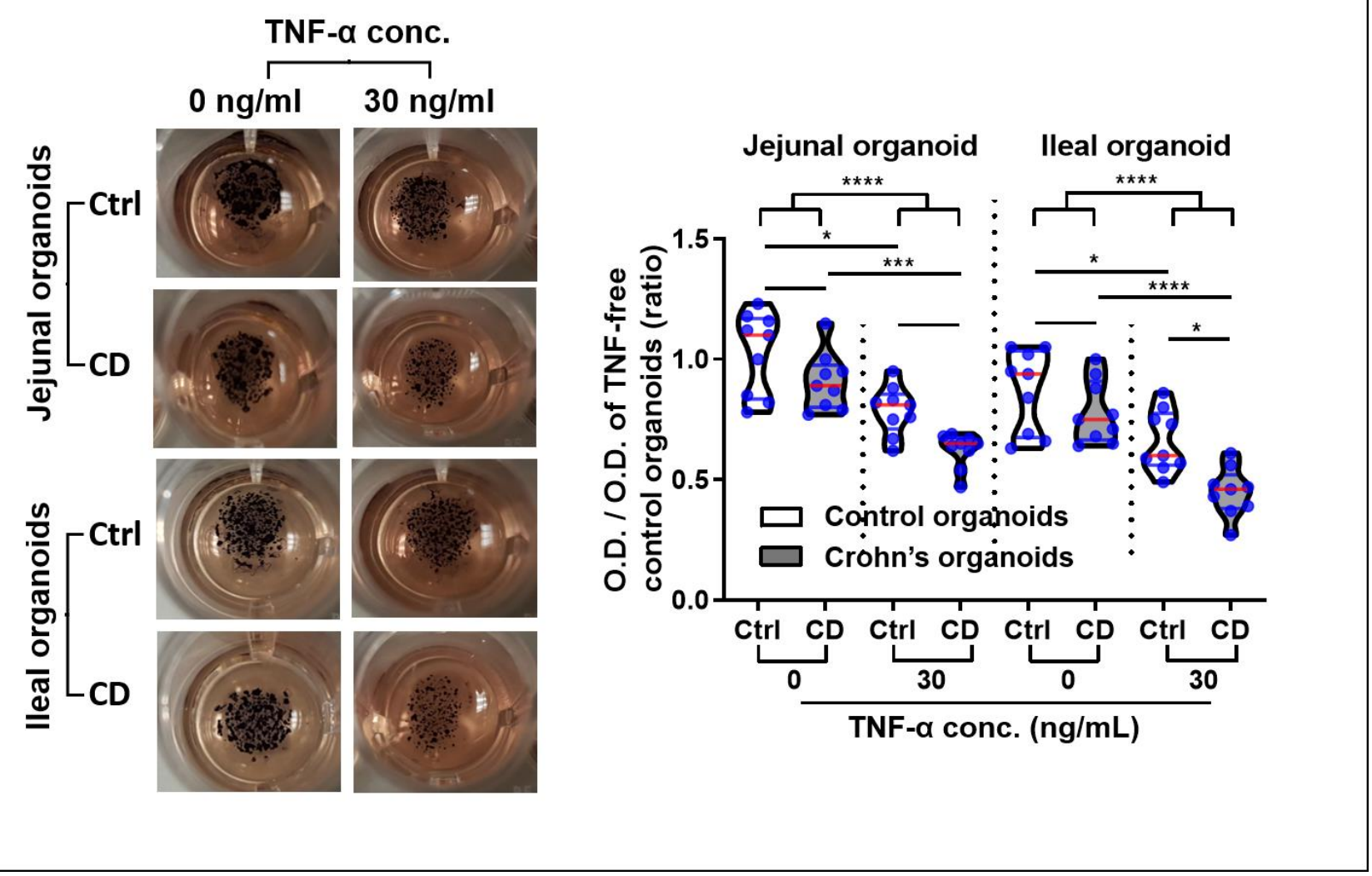

Figure 6. MTT assay for organoid viability in a TNF $\alpha$-enriched condition. MTT assays were performed in triplicate using jejunal and ileal organoids derived from controls and CD patients ( $n=3$ each). The optical density (OD) of organoidcontaining wells is expressed in a ratio based on the values of the TNF $\alpha$-free control organoid-containing wells. Differences were evaluated using ANOVA with Bonferroni's multiple comparison test; ${ }^{*} p<0.05,{ }^{* * *} p<0.001$ and ${ }^{* * * *} p<0.0001$.

Two hours after EdU administration, EdU+ cells were confirmed in the buds, which represented the intestinal crypt (Figure S2). The number of EdU+ cells was higher in TNF $\alpha$ free organoids than in TNF $\alpha$-treated organoids $(91.9 \pm 32.6$ vs. $32.3 \pm 22.2, p<0.001)$. Although there was no significant difference in the number of EdU+ cells between the control and CD patient-derived organoids in the steady-state $(82.7 \pm 33.1$ vs. $102.4 \pm 30.5$, $p=0.653)$, the number of EdU + cells were significantly lower in TNF $\alpha$-treated CD patientderived organoids than in TNF $\alpha$-treated control organoids ( $49.0 \pm 19.1$ vs. $15.6 \pm 7.7$, $p=0.001$, Figure 7). 


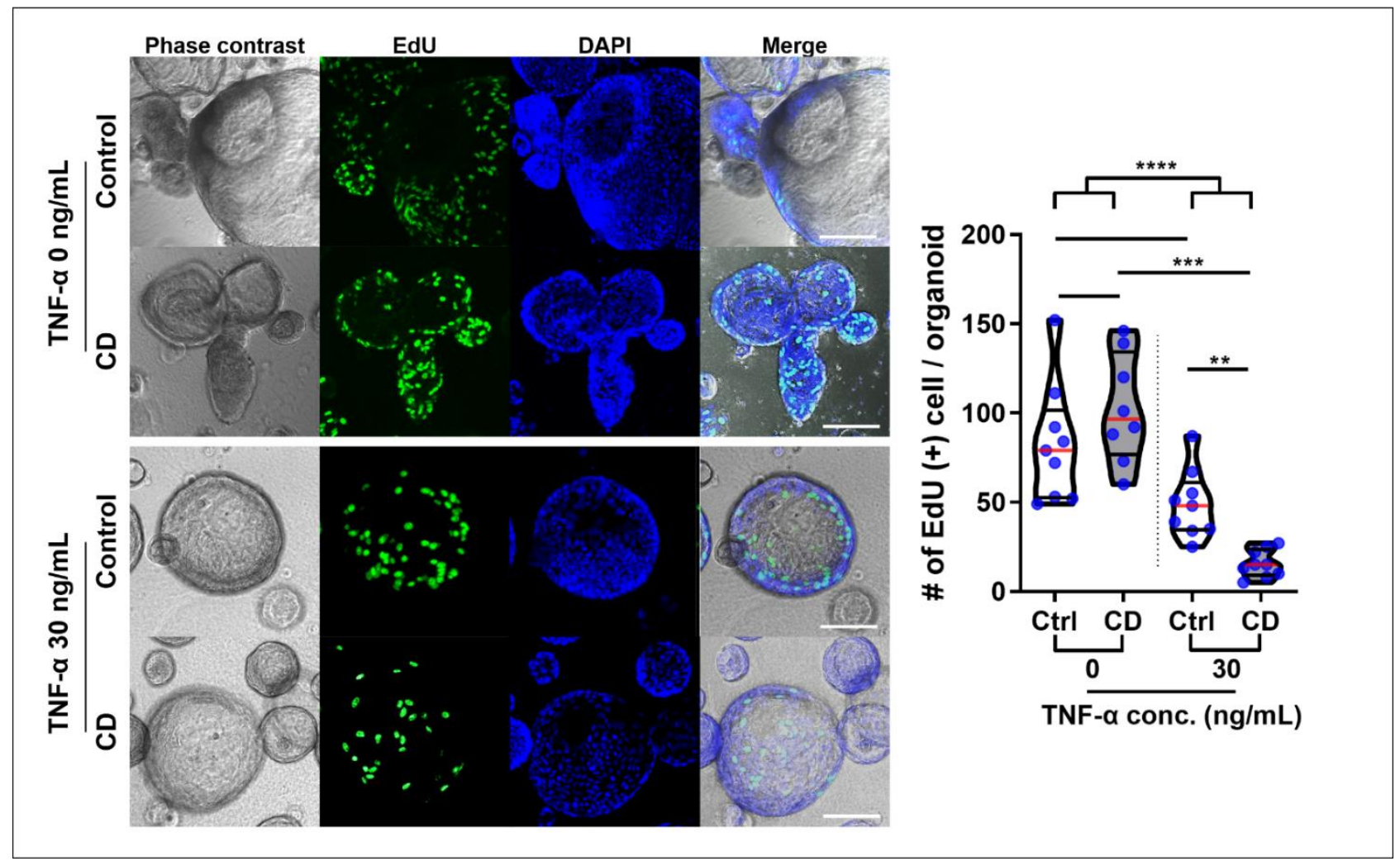

Figure 7. EdU assay for organoid proliferation in a TNF $\alpha$-enriched condition EdU+ cell number was measured $2 \mathrm{~h}$ after EdU administration to 10 organoids from TNF $\alpha$-treated and -free control and CD patient-derived organoids $(n=3$ each). Differences were evaluated using ANOVA and Bonferroni's multiple comparison test; ${ }^{* *} p<0.01,{ }^{* * *} p<0.001$ and $* * * * \quad p<0.0001$.

The wound healing assay showed that the unhealed wound area in TNF $\alpha$-treated CD patient-derived organoids was significantly larger than that in control organoids at $8 \mathrm{~h}$ $(50.5 \% \pm 10.5 \%$ vs. $84.7 \% \pm 12.3 \%, p<0.001), 16 \mathrm{~h}(11.0 \% \pm 4.8 \%$ vs. $64.3 \% \pm 14.0 \%$, $p<0.001)$, and $24 \mathrm{~h}$ after insert removal $(1.7 \% \pm 1.5 \%$ vs. $37.0 \% \pm 8.5 \%, p<0.001)$. There was no significant difference in wound healing between the organoids derived from controls and CD patients. The unhealed wound area at $24 \mathrm{~h}$ was not significantly different between the TNF $\alpha$-free and TNF $\alpha$-treated control organoids $(1.7 \% \pm 1.5 \%$ vs. $16.0 \% \pm 4.6 \%, p=0.257$; ; however, there was a significant difference between the TNF $\alpha$ free and TNF $\alpha$-treated CD patient-derived organoids $(2.3 \% \pm 2.5 \%$ vs. $37.0 \% \pm 8.5 \%$, $p<0.001)$. In addition, the unhealed wound area at $24 \mathrm{~h}$ of TNF $\alpha$-treated CD patientderived organoids was significantly larger than that in TNF $\alpha$-treated control organoids at $24 \mathrm{~h}$ after insert removal $(16.0 \% \pm 4.6 \%$ vs. $37.0 \% \pm 8.5 \%, p=0.044)$. The wound-healing ability of TNF $\alpha$-treated CD patient-derived organoids was significantly lower than that of $\mathrm{TNF} \alpha$-free control and CD patient-derived organoids and TNF $\alpha$-treated control organoids (Figure 8). 


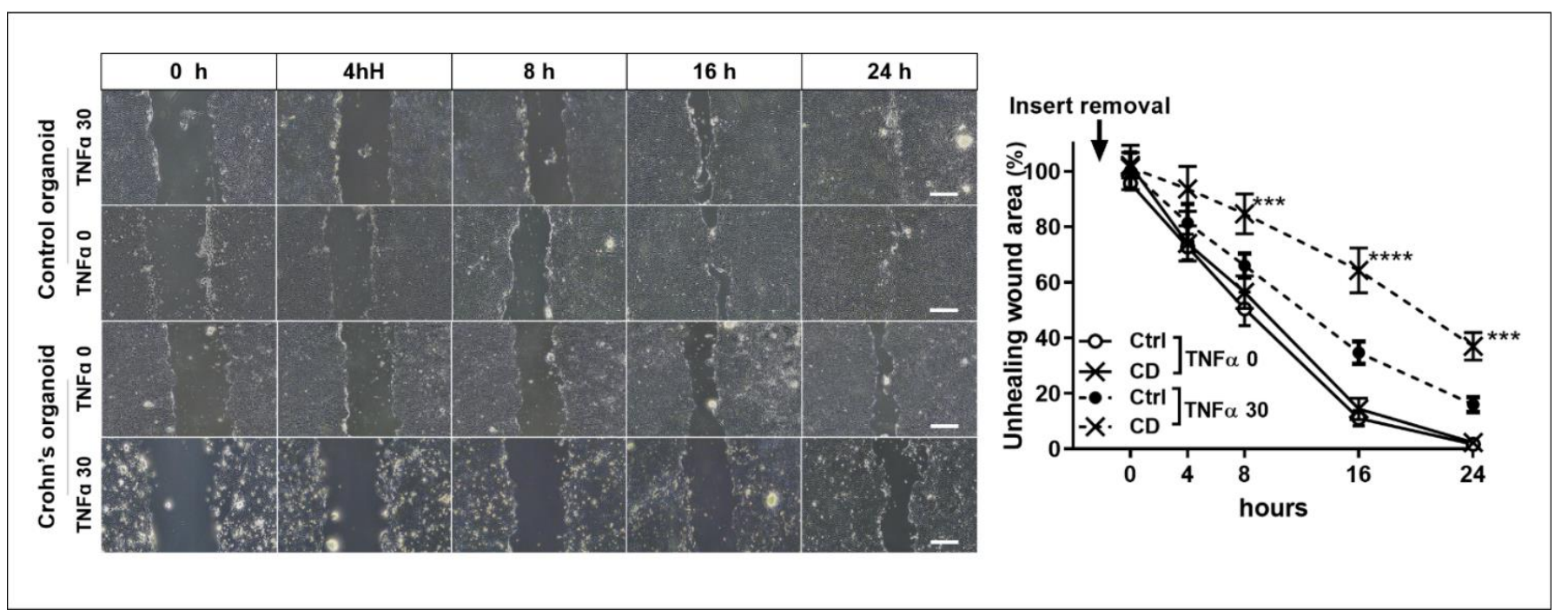

Figure 8. Wound healing assay. Non-healing wound areas in three different areas selected from TNF $\alpha$-free and -treated organoids derived from controls and CD patients were measured ( $n=3$ each). The area of the unhealed wound is expressed in $\%$, based on the observed area of TNF $\alpha$-free control organoids. Differences were evaluated using two-way ANOVA test with Bonferroni's multiple comparison test; ${ }^{* * *} p<0.001$ and ${ }^{* * *} p<0.0001$. Scale bar $=4 \mathrm{~mm}$.

RNA-seq was performed on endoscopic biopsy tissue samples from controls and patients with $C D$ and organoids derived from these samples. The clustering heatmap and principal component analysis identified that the gene expression profile was separated between the endoscopic biopsy tissue samples and the organoids derived from these samples. Furthermore, among the gene expression profile of organoids, there was a clear distinction between the TNF $\alpha$-free and -treated organoids (Figure 9A,B). The epithelial lineage-specific gene expression was evaluated in the tissue samples and organoids derived from these samples (Figure 9C). In endoscopic biopsy tissue samples, the expression of genes associated with the intestinal microbiota (NOD2, DEFA5, DEFA6, PLA2G2A, MUC2, and NARP6) and differentiated cells (enterocytes (ECs): SI, APOC3, ALPI, and APOA1; goblet cells (GCs): MUC2; Paneth cells (PCs): WNT3, ARG2, DLL1, and DLL4; and enteroendocrine cells (EECs): CCK, CHGA, CHGB, and NEUROG3)) was increased in comparison to organoids. The expression of EC markers such as VIL1, KLF5, and KRT5, and GC markers such as TFF3 and MUC13 was upregulated in biopsy tissue samples and TNF $\alpha$ free control organoids (day 6 and 9 organoids). In the TNF $\alpha$-treated control organoids, the expression of ISC markers, such as LGR5, OLFM4, and TNFRSF19, was increased compared with those of TNF $\alpha$-treated CD patient-derived organoids. The alteration of ISC properties can affect cell proliferation and wound healing ability. 


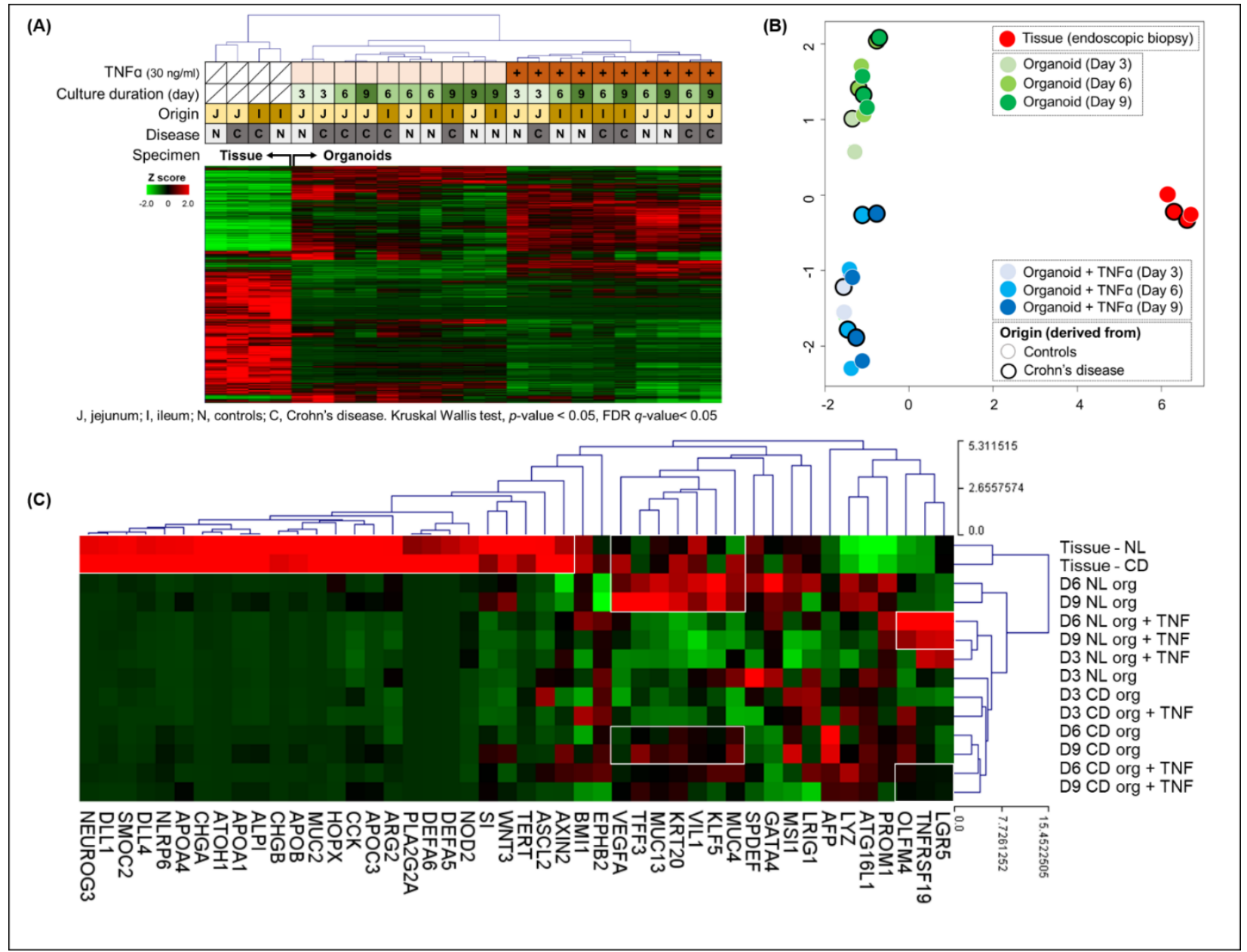

Figure 9. Gene expression profile of the endoscopic biopsy tissue samples from controls and patients with CD and organoids derived from these samples. (A) Clustering heatmap and (B) principal component analysis. The gene expression profile was clearly separated between the endoscopic biopsy tissue samples and the organoids derived from these samples. Among the gene expression profiles of organoids, a clear distinction was noticed between TNF $\alpha$-free and -treated organoids. (C) Epithelial lineage-specific gene expression. The expression of differentiated cell markers was increased in endoscopic tissue biopsy samples. However, the expression of enterocyte markers, such as VIL1, KLF5, and KRT5, and goblet cell markers, such as TFF3 and MUC13, was increased in the biopsy tissue samples, as well as the TNF $\alpha$-free organoids. In the TNF $\alpha$-treated control organoids, the expression of ISC markers, such as LGR5, OLFM4, and TNFRSF19, was increased compared with those of TNF $\alpha$-treated CD patient-derived organoids.

\section{Discussion}

Rapid restoration of epithelial defects following injuries or physiologic damage is indispensable for maintaining gut homeostasis [10]. CD is characterized by chronic and transmural inflammation that can occur along the entire GI tract, but primarily occurs in the small intestine. Nevertheless, the ability for epithelial regeneration and wound healing in patients with CD has not been evaluated, especially by the location in the gastrointestinal tract. To our knowledge, this study is the first to evaluate organoid-forming efficiency using patient-derived organoids in patients with CD according to the location in the GI tract.

Several previous observations suggested that epithelial regeneration and wound healing capacity was impaired in patients with CD. Patients with CD have a higher rate of postoperative complication, in particular anastomotic complications leading to intraabdominal sepsis, than patients without inflammatory status [17]. Exposure to ionizing radiation causes cell death of rapidly proliferating cells, leading to acute or chronic gastrointestinal (GI) toxicity in a dose- and time-dependent manner [18]. Patients with CD 
presented an increased risk of GI toxicity following exposure to therapeutic doses of ionizing radiation [19]. The mutation of CD susceptibility genes, such as NOD2 and ATG16L1, has been associated with ISC dysfunction, leading to impaired epithelial regeneration and wound healing $[20,21]$. IBD patient-derived colonoids showed decreased organoid size and amount of budding, increased cell death and luminal debris, and inverted polarization [22] However, the epithelial regeneration ability of CD patients was not directly evaluated in these previous studies, whereas our study evaluated epithelial regeneration capability in a detailed and direct manner.

This study identified that the organoid-formation ability of CD patient-derived ileal crypts was significantly impaired; this was correlated with the fact that the ileum is the most frequently affected area in CD [23]. These ileal crypts were endoscopically obtained from the uninflamed intestine; however, the effect of pre-existing microscopic inflammation on organoid formation cannot be completely excluded. Long-term cultured CD patientderived organoids were passaged under non-inflammatory culture conditions and showed no significant differences in morphology and culturing behaviors compared to control organoids, which can mimic the mucosal healing in patients with IBD. Mucosal healing is considered to be an ideal therapeutic target for the long-term remission of IBD [3]. However, while patients achieve mucosal healing, several triggers such as inflammatory cytokines and nonsteroidal anti-inflammatory drugs can cause mucosal damage and inflammation. In this study, TNF $\alpha$ was used as a trigger to mimic the inflammatory milieu. TNF $\alpha$ reduced the cell viability and organoid reconstitution ability of intestinal organoids in a dosedependent manner. We identified TNF $30 \mathrm{ng} / \mathrm{mL}$ as an appropriate concentration for the experiment, because the surviving cells showed morphological changes and were sufficient for additional experiments. Previous studies have also suggested that $30 \mathrm{ng} / \mathrm{mL}$ of TNF $\alpha$ may induce cytotoxicity and distinctive cell responses in intestinal organoids $[15,16]$. In a culture condition with $30 \mathrm{ng} / \mathrm{mL}$ of TNF $\alpha$, both jejunal and ileal organoids derived from CD patients showed reduced organoid reconstitution ability, cell viability, cell proliferation, and wound healing capability compared with control organoids.

Previous studies have shown that wound healing is accomplished through epithelial restitution, ISC proliferation, and differentiation, although these wound healing processes overlap [17-19]. This study evaluated the epithelial restitution using wound healing assays and achieving organoid cell proliferation using organoid reconstitution, MTT, and EdU assay. Post-injury wound healing is regulated by a wide range of regulatory factors, including cytokines, growth factors, adhesion molecules, and phospholipids [10,20]. Inflammatory processes have especially been thought to interfere with epithelial cell migration and proliferation, and thus modulate intestinal epithelial healing [21]. However, in this study, RNA-seq identified that the expression of active ISC markers such as LGR5, OLFM4, and TNFRSF19 was decreased in CD patient-derived organoids compared with the control organoids under a TNF $\alpha$-enriched condition. Under TNF $\alpha$-enriched culture conditions, the ISC population may be expanded for reparative proliferation to replace lost cells with $\mathrm{TNF} \alpha$-induced cytotoxicity. However, alterations in ISC properties in organoids derived from CD patients in response to TNF $\alpha$ may affect ISC proliferation, and subsequently the wound healing ability can be impaired. Previous single-cell RNA-seq also identified alterations in ISC properties in CD patient-derived small intestinal organoids [24], which supports our RNA-seq results. Based on our results, inflammatory processes can particularly interfere with ISC proliferation, and therefore modulate intestinal epithelial wound healing.

A limitation of our study is that the organoid culture system does not reflect the effects of intestinal microbiota, dietary components, and the mucosal immune system. The intestinal microbiota and dietary components contribute to the fine-tuning of ISC survival and differentiation [25]. In addition, only ileal crypts of $C D$ showed a significant reduction in organoid-formation efficiency compared with those of controls. As enteroscopy was usually performed for the diagnosis and treatment of $C D$ via the per-anal route, ileal sampling was relatively convenient and capable of obtaining a sufficient sample size but 
duodenal and jejunal sampling were not. The lack of significance of the duodenal, jejunal, and colonic organoid reconstitution rates might be attributed to the small sample size.

The aim of this study was to evaluate whether the epithelial regeneration ability is impaired in patients with $\mathrm{CD}$. The organoid-forming efficiency of the ileal crypts in patients with $C D$ was reduced compared with those of the control subjects. When TNF $\alpha$ was used as a trigger to mimic the inflammatory milieu, the epithelial regeneration and wound healing ability was decreased more noticeably in CD patient-derived organoids compared to in control organoids. Our findings suggest the epithelial regeneration ability was impaired in patients with CD. Clinical trials are unable to settle this issue due to ethical reasons and clinical heterogeneity, while our results indicated that the epithelial regenerative ability is impaired in patients with CD, especially in TNF $\alpha$-enriched conditions. Impaired epithelial regeneration results in defective mucosal integrity and sustained intestinal inflammation, leading to ulcers, fibrosis, and fistulas, which are the main indications for surgery in patients with CD. Mucosal healing can be regarded as epithelial regeneration after injury of the intestinal lining. Mucosal healing is considered a therapeutic target of IBD [26]. To improve mucosal healing in patients with $\mathrm{CD}$, additional treatment options to promote epithelial regeneration and wound healing ability should be explored and developed.

\section{Materials and Methods}

\subsection{Sampling}

To establish intestinal organoids, we used human intestinal tissue from controls and patients with CD using biopsy forceps during single-balloon enteroscopy at the Samsung Medical Center, Seoul Korea, between November 2016 and December 2018. At least four biopsy samples were obtained from mucosal tissues in the jejunum $(100-150 \mathrm{~cm}$ distal of the ligamentum of Treitz), ileum (50-100 cm proximal to the ileocecal valve), and colon (transverse colon). Patients with CD were diagnosed according to the guidelines [27]. In patients with $C D$, biopsies were performed at least $5 \mathrm{~cm}$ away from the ulcers. All samples were acquired with informed consent. This study was approved by the institutional ethical committee of the Samsung Medical Center (IRB No. 2016-02-022, approval date: 10/12/2016).

\subsection{Crypt Isolation from the Biopsy Specimens}

Endoscopic biopsy samples were incubated in PBS with $10 \mathrm{mM}$ ethylenediaminetetraacetic acid (Thermo Fischer Scientific, San Jose, CA, USA) and $1 \mathrm{mM}$ dithiothreitol (Thermo Fischer Scientific) at $4{ }^{\circ} \mathrm{C}$ for $30 \mathrm{~min}$, and then vortexed for 30-120 s. The supernatant containing crypts was filtered through 70- $\mu$ m cell strainers (Corning, Bedford, MA, USA) and suspended in basal medium (advanced Dulbecco's modified Eagle's medium (DMEM)/F12 (Thermo Fischer Scientific) supplemented with antibiotic-antimycotic solution (Thermo Fischer Scientific), HEPES (Thermo Fischer Scientific), GlutaMAX (Thermo Fischer Scientific), N2 (Thermo Fischer Scientific), B27 (Thermo Fischer Scientific), and $\mathrm{N}$-acetylcysteine (Sigma-Aldrich, St. Louis, MO, USA).

\subsection{Three-Dimensional (3D) Intestinal Crypt Culture}

Isolated crypts were resuspended in Matrigel (Corning) and plated in 48-well culture plates (Corning). After incubation at $37^{\circ} \mathrm{C}$ for $15 \mathrm{~min}, 250 \mu \mathrm{L}$ of maintenance medium (50\% Wnt3a-conditioned medium (ATCC\#CRL-2647, Manassas, VA, USA) and 50\% of $2 \times$ basal medium, supplemented with recombinant human EGF (Sigma-Aldrich), recombinant human noggin (R\&D Systems, Minneapolis, MN, USA), recombinant human R-spondin1 (PeproTech, Cranbury, NJ, USA), nicotinamide (Sigma-Aldrich), p160ROCK inhibitor (Selleck Chemicals, Houston, TX, USA), p38 MAP kinase inhibitor (SB202190, SigmaAldrich), and Prostaglandin E2 (PGE2, Cayman Chemical, Ann Arbor, MI, USA), were added to the wells. A GSK3 inhibitor (Stemgent, Cambridge, MA, USA) was added to the medium during the first 2 days. 
Depending on their shape, organoids can be classified into spheroids and enteroids. Spheroids are defined as round- or oval-shaped organoids with a thin wall composed of a single layer of undifferentiated cells. Enteroids are defined by the presence of visually sharp borders (buddings) along their basolateral (anti-luminal) side or irregularly thickened walls, which consist of all components of epithelial cells [28] (Figure 10).

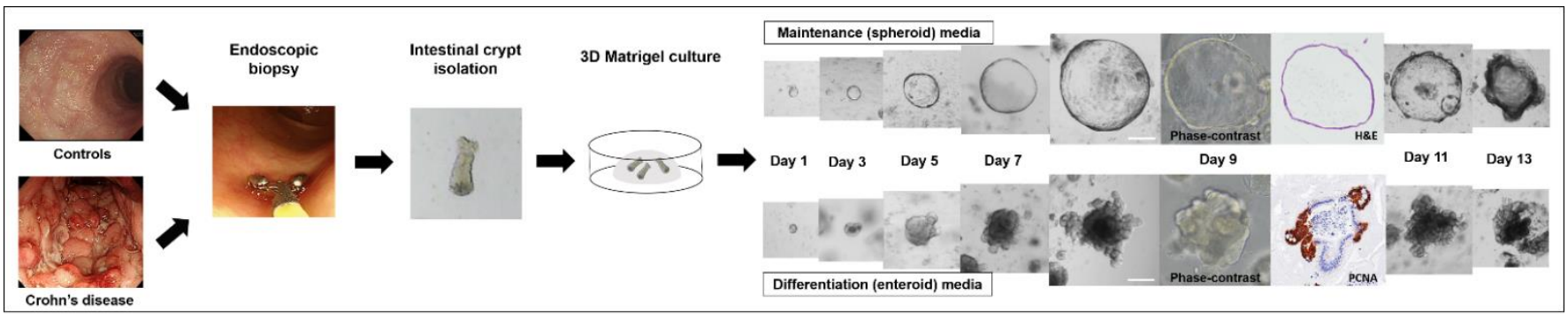

Figure 10. Flow diagram of the patient-derived intestinal organoid model. Scale bar $=100 \mu \mathrm{m}$.

\subsection{Organoid-Forming Efficiency}

One hundred crypts obtained from control subjects and CD patients were plated in $25 \mu \mathrm{L}$ of Matrigel in maintenance medium. The organoid-forming efficiency was calculated as the percentage of viable organoids per 100 intestinal crypts.

\subsection{Organoid Subculture, Maintenance, and Differentiation}

After 7 days of the culture process, the organoids were mechanically disrupted and suspended in cell dissociation buffer (Thermo Fischer Scientific). Single cells and small cell clusters were resuspended in Matrigel and plated in 48-well culture plates (Corning). The medium was changed every 2 days, and the organoids were passaged at a ratio of 1:2-1:4 on day 7. After 6 passages, most organoids in the maintenance medium formed uniform spheroids and could be subcultured stably for a long time in vitro.

To recreate the physiological parameters of the intestinal epithelium, the spheroids were cultured in a differentiation medium (maintenance medium without Wnt3A conditional medium, SB202190, nicotinamide, and PGE2). The differentiation medium was changed every 2 days and enteroids were grown for 7-12 days.

\subsection{Organoid Reconstitution Assay}

After more than six passages, the organoids were mechanically disrupted and suspended in the cell dissociation buffer. Single cells were resuspended in Matrigel and plated in 48-well culture plates. The intestinal organoids were cultured in maintenance medium for 2 days to obtain a stable number of organoids before inducing organoid differentiation; then, the maintenance medium was changed to a differentiation medium every 2 days. Different concentrations of human recombinant TNF $\alpha$ (R\&D Systems) were added to the culture medium every $24 \mathrm{~h}$. The organoid reconstitution rate was calculated as the percentage of the number of viable organoids on day 9-10 proportional to the number of viable cells on day 2.

\subsection{3-(4,5-Dimethylthiazolyl-2)-2,5-diphenyltetrazolium Bromide (MTT) Assay}

Ten microliters of MTT (Sigma-Aldrich) was added to each well of the culture plates and incubated for $3 \mathrm{~h}$ until purple precipitates were visible. After $100 \mu \mathrm{L}$ of detergent reagent was added, the organoids were incubated at room temperature in the dark for $2 \mathrm{~h}$, prior to recording the absorbance at $570 \mathrm{~nm}$. The optical density (OD) of organoidcontaining wells is expressed in a ratio based on the values of TNF $\alpha$-free control organoidcontaining wells. 


\subsection{5-Ethynyl-2'-Deoxyuridine (EdU) Assay}

Two hundred micrograms of EdU (Abcam, Cambridge, UK) was added to the culture medium $2 \mathrm{~h}$ before fixation with cold $4 \%$ paraformaldehyde (Biosesang. Seongnam-si, South Korea). Incorporation of EdU into DNA was detected using a Click-iT ${ }^{\mathrm{TM}} \mathrm{EdU}$ Alexa Fluor ${ }^{\circledR} 488$ imaging kit (Thermo Fischer Scientific). The number of EdU $(+)$ cells was measured in 10 organoids (size $>100 \mu \mathrm{m}$ ) selected from the TNF $\alpha$-free and -treated enteroids derived from the controls and $\mathrm{CD}$ patients.

\subsection{Wound Healing Assay}

3D cultured organoids were digested into single cells using TrypLE Express (Thermo Fischer Scientific) and $5 \times 10^{4}$ cells were seeded into 24-well plates containing CytoSelect ${ }^{\mathrm{TM}}$ 24-Well Wound Healing Assay inserts (Cell Biolabs, San Diego, CA, USA) [29]. Organoid monolayers were cultured in maintenance medium until confluence was reached. The inserts were then carefully removed to produce $0.9 \mathrm{~mm}$ diameter wounds, and fresh differentiating medium was added to each well. The area of the unhealed wound was measured in three different areas. The area of the unhealed wound is expressed in $\%$, based on the observed area of $\mathrm{TNF} \alpha$-free control organoids.

\subsection{Real-Time Quantitative Reverse Transcription Polymerase Chain Reaction (qPCR)}

One-step qPCR was performed using One Step PrimeScript ${ }^{\mathrm{TM}}$ III RT-qPCR Mix (Takara, Kusatsu, Japan) with primers as follows: LGR5 primer (Forward: $5^{\prime}$-aactttggcattgtggaagg$3^{\prime}$, Reverse: $5^{\prime}$-acacattgggggtaggaaca-3'), BMI1 primer (Forward: $5^{\prime}$-cgtgtattgttcgttacctgga$3^{\prime}$, Reverse: $5^{\prime}$-ttcagtagtggtctggtcttgt- $3^{\prime}$ ), ATOH1 primer (Forward: $5^{\prime}$-cagctgcgcaatgttatccc$3^{\prime}$, Reverse: $5^{\prime}$-ttgtagcagctcggacaagg- $3^{\prime}$ ), and HES1 primer (Forward: $5^{\prime}$-tttcctcattcccaacgggg$3^{\prime}$, Reverse: $5^{\prime}$-ctggaaggtgacactgcgtt-3').

\subsection{RNA Sequencing}

RNA sequencing was conducted on endoscopic biopsy tissue samples from controls and patients with $C D$ and organoids derived from these samples. Total RNA was extracted from intestinal organoids using an RNeasy Mini Kit (QIAGEN, Hilden, Germany). RNA was isolated from endoscopic biopsy tissue samples from controls $(n=2)$ and patients with $\mathrm{CD}(n=2$, non-inflamed area, during infliximab maintenance treatment). In addition, organoid culture was conducted with intestinal crypts derived from these specimens. Organoids were sub-cultured for least 6 passages. RNA was isolated from the organoids on day 3 , day 6 , and day 9 , and organoids were treated with $\mathrm{TNF} \alpha 30 \mathrm{ng} / \mathrm{mL}$ on day 3 , day 6 , and day 9. RNA sequencing was performed using total RNA samples with $>10 \mu \mathrm{g}$ of RNA and an integrity number $>8$. The libraries were constructed for whole-transcriptome sequencing using the TruSeq RNA Sample Preparation Kit v2 (Illumina, San Diego, CA, USA) and sequenced using the 100-bp paired-end mode of the TruSeq Rapid PE Cluster Kit and the TruSeq Rapid SBS Kit (Illumina).

Reads from files in the FASTQ format were mapped to the hg19 human reference genome using HISAT 2.2.0, with default parameters (https: / / daehwankimlab.github.io/ hisat2/, accessed on 18 May 2021). Raw read counts mapped to genes were measured using the BAM format file in HTSeq version 0.12 .3 (https://htseq.readthedocs.io/, accessed on 18 May 2021), to quantify transcript abundance. The coding genes were selected, and raw read counts were normalized to the trimmed mean of M-values. Differential expression analysis of RNA-seq experiments was conducted with edgeR (version 3.28.1). Unsupervised hierarchical clustering analysis with the Euclidean distance and complete linkage algorithm was used to create a heatmap with the associated dendrogram.

\subsection{Availability of Data and Materials}

The datasets presented in this study can be found in GEO (accession number = GSE173294, https://www.ncbi.nlm.nih.gov/geo/query/acc.cgi?acc=GSE173294, accessed on 18 May 2021). 
Supplementary Materials: The following are available online at https://www.mdpi.com/article/10 $.3390 / \mathrm{ijms} 22116013 / \mathrm{s} 1$.

Author Contributions: Conceptualization, C.L. and S.-N.H.; Methodology, C.L. and S.-N.H.; Validation, C.L. and S.-N.H.; Investigation, C.L., E.-R.K., D.-K.C., Y.-H.K. and S.-N.H.; Resources, E.-R.K., D.-K.C., Y.-H.K. and S.-N.H.; Data Curation: C.L. and S.-N.H.; Writing-Original Draft, C.L. and S.-N.H.; Writing—Review \& Editing, E.-R.K., D.-K.C., Y.-H.K. and S.-N.H.; Supervision, Y.-H.K.; Project Administration, S.-N.H.; Funding Acquisition, C.L. and S.-N.H. All authors have read and agreed to the published version of the manuscript.

Funding: This work was supported by the National Research Foundation of Korea (NRF) grant funded by the Korea government (MSIP) (NRF-2018R1D1A1B07048376, NRF-2019R1I1A1A01062205, and NRF-2019R1A2C2010404). This funding source had no role in the design of this study and will not have any role during its execution, analyses, interpretation of the data and decision to submit results.

Institutional Review Board Statement: The study was conducted according to the guidelines of the Declaration of Helsinki, and approved by the Institutional Ethical Committee of Samsung Medical Center (IRB No. 2016-02-022).

Informed Consent Statement: Informed consent was obtained from all subjects involved in the study.

Data Availability Statement: The datasets presented in this study can be found in GEO (accession number = GSE173294, https: / / www.ncbi.nlm.nih.gov/geo/query/acc.cgi?acc=GSE173294, accessed on 18 May 2021).

Acknowledgments: I would like to thank Martín G. Martín, (Department of Pediatrics, Mattel Children's Hospital and the David Geffen School of Medicine, University of California Los Angeles), Matthias Stelzner (Department of Surgery, Veterans Affairs Greater Los Angeles Healthcare System), and James C. Y. Dunn (Division of Pediatric Surgery, Department of Surgery, Stanford University) who taught and trained me in construct intestinal organoid models, despite of their busy schedules.

Conflicts of Interest: The authors declare no conflict of interest.

\section{References}

1. Fordham, R.P.; Yui, S.; Hannan, N.R.; Soendergaard, C.; Madgwick, A.; Schweiger, P.J.; Nielsen, O.H.; Vallier, L.; Pedersen, R.A.; Nakamura, T.; et al. Transplantation of expanded fetal intestinal progenitors contributes to colon regeneration after injury. Cell Stem Cell 2013, 13, 734-744. [CrossRef] [PubMed]

2. Metcalfe, C.; Kljavin, N.M.; Ybarra, R.; de Sauvage, F.J. Lgr5+ stem cells are indispensable for radiation-induced intestinal regeneration. Cell Stem Cell 2014, 14, 149-159. [CrossRef]

3. Torres, J.; Mehandru, S.; Colombel, J.F.; Peyrin-Biroulet, L. Crohn's disease. Lancet 2017, 389, 1741-1755. [CrossRef]

4. Mizoguchi, E.; Low, D.; Ezaki, Y.; Okada, T. Recent updates on the basic mechanisms and pathogenesis of inflammatory bowel diseases in experimental animal models. Intest. Res. 2020, 18, 151-167. [CrossRef] [PubMed]

5. Rhim, J.S. Development of human cell lines from multiple organs. Ann. N. Y. Acad. Sci. 2000, 919, 16-25. [CrossRef]

6. Kim, J.; Koo, B.K.; Knoblich, J.A. Human organoids: Model systems for human biology and medicine. Nat. Rev. Mol. Cell Biol. 2020, 1-14. [CrossRef]

7. Sato, T.; Vries, R.G.; Snippert, H.J.; van de Wetering, M.; Barker, N.; Stange, D.E.; van Es, J.H.; Abo, A.; Kujala, P.; Peters, P.J.; et al. Single Lgr5 stem cells build crypt-villus structures in vitro without a mesenchymal niche. Nature 2009, 459, 262-265. [CrossRef]

8. Sato, T.; Stange, D.E.; Ferrante, M.; Vries, R.G.; Van Es, J.H.; Van den Brink, S.; Van Houdt, W.J.; Pronk, A.; Van Gorp, J.; Siersema, P.D.; et al. Long-term expansion of epithelial organoids from human colon, adenoma, adenocarcinoma, and Barrett's epithelium. Gastroenterology 2011, 141, 1762-1772. [CrossRef] [PubMed]

9. Lei, N.Y.; Jabaji, Z.; Wang, J.; Joshi, V.S.; Brinkley, G.J.; Khalil, H.; Wang, F.; Jaroszewicz, A.; Pellegrini, M.; Li, L.; et al. Intestinal subepithelial myofibroblasts support the growth of intestinal epithelial stem cells. PLoS ONE 2014, 9, e84651. [CrossRef]

10. Sturm, A.; Dignass, A.U. Epithelial restitution and wound healing in inflammatory bowel disease. World J. Gastroenterol. 2008, 14, 348-353. [CrossRef] [PubMed]

11. Braegger, C.P.; Nicholls, S.; Murch, S.H.; Stephens, S.; MacDonald, T.T. Tumour necrosis factor alpha in stool as a marker of intestinal inflammation. Lancet 1992, 339, 89-91. [CrossRef]

12. Sedger, L.M.; McDermott, M.F. TNF and TNF-receptors: From mediators of cell death and inflammation to therapeutic giantsPast, present and future. Cytokine Growth Factor Rev. 2014, 25, 453-472. [CrossRef]

13. Delgado, M.E.; Brunner, T. The many faces of tumor necrosis factor signaling in the intestinal epithelium. Genes Immun. 2019, 20, 609-626. [CrossRef] [PubMed] 
14. Klenske, E.; Bojarski, C.; Waldner, M.; Rath, T.; Neurath, M.F.; Atreya, R. Targeting mucosal healing in Crohn's disease: What the clinician needs to know. Therap. Adv. Gastroenterol. 2019, 12. [CrossRef] [PubMed]

15. Kim, Y.S.; Morgan, M.J.; Choksi, S.; Liu, Z.G. TNF-induced activation of the Nox1 NADPH oxidase and its role in the induction of necrotic cell death. Mol. Cell 2007, 26, 675-687. [CrossRef] [PubMed]

16. Csiszar, A.; Smith, K.E.; Koller, A.; Kaley, G.; Edwards, J.G.; Ungvari, Z. Regulation of bone morphogenetic protein-2 expression in endothelial cells: Role of nuclear factor- $\mathrm{kB}$ activation by tumor necrosis factor- $\alpha, \mathrm{H}_{2} \mathrm{O}_{2}$, and high intravascular pressure. Circulation 2005, 111, 2364-2372. [CrossRef] [PubMed]

17. Nusrat, A.; Delp, C.; Madara, J.L. Intestinal epithelial restitution. Characterization of a cell culture model and mapping of cytoskeletal elements in migrating cells. J. Clin. Investig. 1992, 89, 1501-1511. [CrossRef]

18. Moore, R.; Carlson, S.; Madara, J.L. Rapid barrier restitution in an in vitro model of intestinal epithelial injury. Lab. Investig. 1989, 60, 237-244.

19. Feil, W.; Wenzl, E.; Vattay, P.; Starlinger, M.; Sogukoglu, T.; Schiessel, R. Repair of rabbit duodenal mucosa after acid injury in vivo and in vitro. Gastroenterology 1987, 92, 1973-1986. [CrossRef]

20. Okamoto, R.; Watanabe, M. Molecular and clinical basis for the regeneration of human gastrointestinal epithelia. J. Gastroenterol. 2004, 39, 1-6. [CrossRef]

21. Rachmilewitz, D.; Modigliani, R.; Podolsky, D.K.; Sachar, D.B.; Tozun, N. VI International Symposium on Inflammatory Bowel Diseases, Proceedings of the Falk Symposium 123, Istanbul, Turkey, 3-5 September 2001; Kluwer Academic Publishers: Dordecht, The Netherlands; Boston, MA, USA, 2002.

22. D'Aldebert, E.; Quaranta, M.; Sébert, M.; Bonnet, D.; Kirzin, S.; Portier, G.; Duffas, J.P.; Chabot, S.; Lluel, P.; Allart, S.; et al. Characterization of Human Colon Organoids from Inflammatory Bowel Disease Patients. Front. Cell Dev. Biol. $2020,8,363$. [CrossRef] [PubMed]

23. Crohn, B.B. Regional Ileitis. Postgrad. Med. 1965, 38, 276-281. [CrossRef] [PubMed]

24. Suzuki, K.; Murano, T.; Shimizu, H.; Ito, G.; Nakata, T.; Fujii, S.; Ishibashi, F.; Kawamoto, A.; Anzai, S.; Kuno, R.; et al. Single cell analysis of Crohn's disease patient-derived small intestinal organoids reveals disease activity-dependent modification of stem cell properties. J. Gastroenterol. 2018, 53, 1035-1047. [CrossRef] [PubMed]

25. Delgado, M.E.; Grabinger, T.; Brunner, T. Cell death at the intestinal epithelial front line. FEBS J. 2016, 283, 2701-2719. [CrossRef]

26. Watanabe, K. Clinical management for small bowel of Crohn's disease in the treat-to-target era: Now is the time to optimize treatment based on the dominant lesion. Intest. Res. 2020, 18, 347-354. [CrossRef] [PubMed]

27. Ye, B.D.; Jang, B.I.; Jeen, Y.T.; Lee, K.M.; Kim, J.S.; Yang, S.K. Diagnostic guideline of Crohn's disease. Korean J. Gastroenterol. 2009, 53, 161-176. [PubMed]

28. Stelzner, M.; Helmrath, M.; Dunn, J.C.; Henning, S.J.; Houchen, C.W.; Kuo, C.; Lynch, J.; Li, L.; Magness, S.T.; Martin, M.G.; et al. A nomenclature for intestinal in vitro cultures. Am. J. Physiol. Gastrointest. Liver Physiol. 2012, 302, G1359-G1363. [CrossRef] [PubMed]

29. Lin, J.Y.; Lo, K.Y.; Sun, Y.S. A microfluidics-based wound-healing assay for studying the effects of shear stresses, wound widths, and chemicals on the wound-healing process. Sci. Rep. 2019, 9, 20016. [CrossRef] 